sentially the same normalized value of opticalfrequency conductivity. In our model only bandwidth differences distinguish ionic from covalent crystals. Neither the energy gap nor the internuclear spacing enter into the computation of $\sigma$. The bandwidth parameters $b_{c}$ and $b_{i}$ measure the width of those parts of the conduction and valence bands which play important roles in determining crystal structure. For the more covalent crystals Phillips ${ }^{12}$ has correlated crystal structure with a dielectrically defined bond ionicity. Here we have found from a spectroscopic analysis of covalent and ionic crystals that the $N_{\mathrm{c}}$ and $Z_{\mathrm{a}}$ of Pauling's classical resonating-bond theory ${ }^{13}$ play an important role. In both cases the usual picture of crystal structure as determined only by the energies of occupied valence states is discarded in favor of relationships between structure and the optical spectrum.

We wish to thank J. C. Phillips and J. A. Van Vechten for stimulating discussions and helpful comments on the manuscript.

${ }^{1}$ J. C. Phillips, Phys. Rev. Letters 20, 550 (1968)。

${ }^{2}$ J. C. Phillips and J. A. Van Vechten, Phys. Rev. Letters 22, 705 (1969).

${ }^{3}$ J. A. Van Vechten, Phys. Rev. 182, 891 (1969).

${ }^{4} \mathrm{~S}$. H. Wemple and M. DiDomenico, Jr., to be pub- lished; for data on some oxides see M. DiDomenico, Jr., and S. H. Wemple, J. Appl. Phys。 40, 720 (1969). ${ }^{5}$ We have excluded from Table I all small-band-gap semiconductors since it is difficult to determine with sufficient accuracy the free-carrier and/or photoionization contribution to the observed refractive-index dispersion for such materials. The results tabulated for Ge may in fact be influenced by such extrinsic effects.

${ }^{6}$ In magnetic transition-metal compounds we would expect weaker oscillator strength for transitions from the occupied $d$ or $f$ orbitals. For example, in EuO $\mathcal{E}_{\mathrm{d}}$ $=9 \mathrm{eV}$ rather than approximately $25 \mathrm{eV}$ as observed in 6 -coordinated nonmagnetic oxides. In complex crystals containing anion radicals we find, for example, that $\mathcal{E}_{\mathrm{d}} \approx 16 \mathrm{eV}$ for several phosphates and $\mathcal{E}_{\mathrm{d}} \approx 20 \mathrm{eV}$ for a group of iodates and carbonates.

${ }^{7}$ Based on available refractive-index data the $\beta$ values for $\mathrm{LiF}$ and $\mathrm{AgCl}$ fall slightly outside the limits given for $\beta_{\mathbf{i}}$.

${ }^{8}$ The $\beta$ value for Ge lies slightly below the lower bound given for $\beta_{\mathrm{c}}$. This may be due to a small freecarrier contribution to the refractive index data.

${ }^{9}$ S. Kurtin, T. C. McGill, and C. A. Mead, Phys. Rev. Letters 22, 1433 (1969).

${ }^{10}$ D. R. Penn, Phys。Rev. 128, 2093 (1962).

${ }^{11}$ We thank J. A. Van Vechten for providing us with the Penn-model $\epsilon_{2}$ spectrum.

${ }^{12}$ J. C. Phillips, Chem. Phys. Letters 3 , 286 (1969).

${ }^{13} \mathrm{~L}$. Pauling, The Nature of the Chemical Bond (Cornell University Press, New York 1960).

\title{
REFLECTION SPECTRUM OF SOLID ARGON IN THE VACUUM ULTRAVIOLET*
}

\author{
R. Haensel and G. Keitel
}

Physikalisches Staatsinstitut, II. Institut für Experimentalphysik der Universität Hamburg, Hamburg, Germany and

E. E. Koch and M. Skibowski

Sektion Physik der Universität München, München, Germany

and

P. Schreiber

Deutsches Elektronen-Synchrotron, Hamburg, Germany

(Received 13 October 1969)

\begin{abstract}
The reflectance of solid Ar has been measured at $20^{\circ} \mathrm{K}$ for an angle of incidence of $15^{\circ}$ in the photon energy range from 10 to $30 \mathrm{eV}$ using the synchrotron radiation of DESY. The reflectance data reveal a spin-orbit-split exciton series with sharp maxima converging to about $14 \mathrm{eV}$ together with broader peaks above $14 \mathrm{eV}$ due to transitions between the valence and conduction band. The results are compared with the absorption spectrum associated with the $2 p$ core levels.
\end{abstract}

Several optical ${ }^{1,2}$ and electron-energy-loss ${ }^{3}$ measurements have been performed on solid Ar in order to study its electronic transitions from the valence band. These investigations were confined to the spectral region below $14 \mathrm{eV}$. They were made with limited resolution so that the existence of an exciton series converging to the band gap could not clearly be proved. Thus these measurements led to some uncertainty in the determination of the series limit. ${ }^{4}$ 


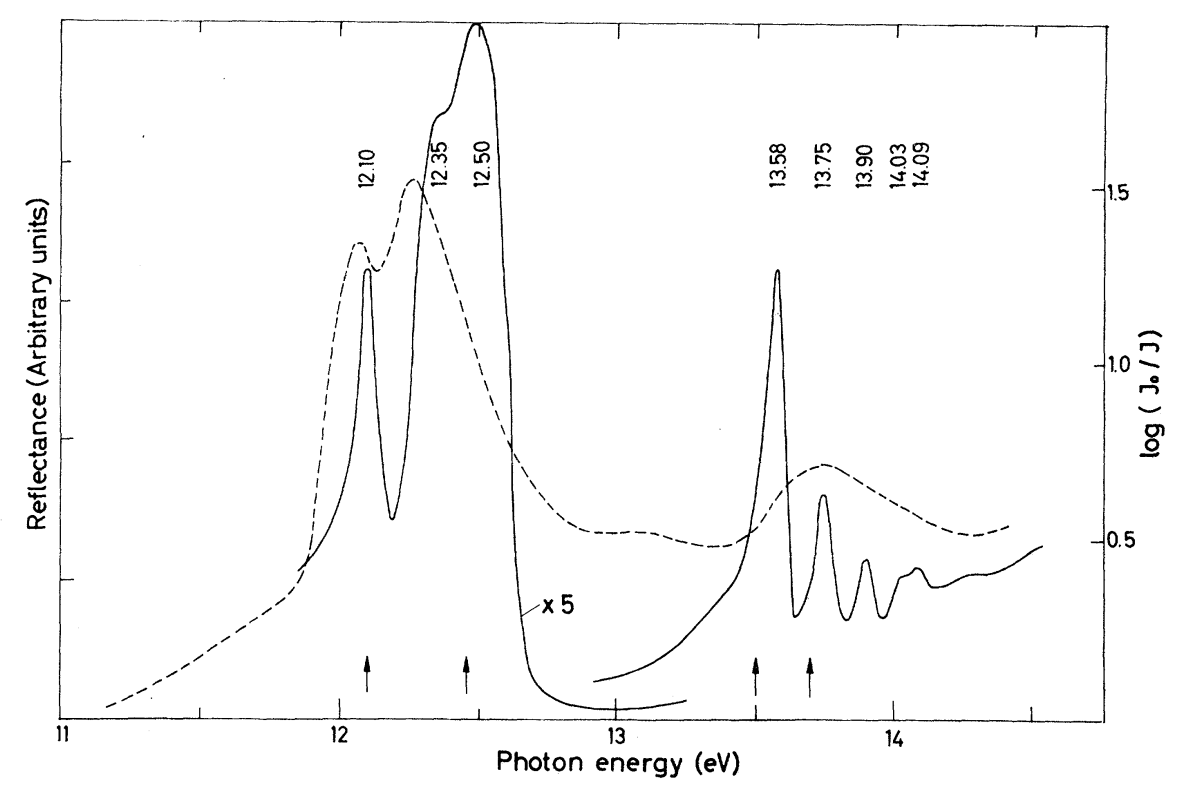

FIG. 1. Reflectance of solid Ar at $20^{\circ} \mathrm{K}$ for an angle of incidence of $15^{\circ}$ between 11 and $14.5 \mathrm{eV}$ (solid line). The dashed line gives Baldini's absorption curve (Ref. 2), right ordinate. Arrows indicate the energy positions of electron-energy-loss peaks obtained by Bostanjoglo and Schmidt (Ref.3).

We have measured the reflectance of solid Ar at $20^{\circ} \mathrm{K}$ for an angle of incidence of $15^{\circ}$ in the energy range 10 to $30 \mathrm{eV}$ with a resolution of $2 \AA$. The synchrotron radiation of the DESY $7.5-\mathrm{GeV}$ electron synchrotron ${ }^{5}$ was monochromatized by a normal-incidence monochromator in a modified Wadsworth mounting. ${ }^{6}$ The Ar was evaporated as a thin film of unknown thickness onto a $\mathrm{KCl}$ single crystal and a glass plate, cooled in a He cryostat.
No significant influence of the two substrates on the reflectance could be observed. The light reflected from the Ar surface was detected with an open photomultiplier (Bendix M 306). Cryostat and multiplier were mounted into a modified commercial ultrahigh vacuum system. Outside the cooled cryostat the chamber pressure was $5 \times 10^{-8}$ Torr.

Figures 1 and 2 show the results of our mea-

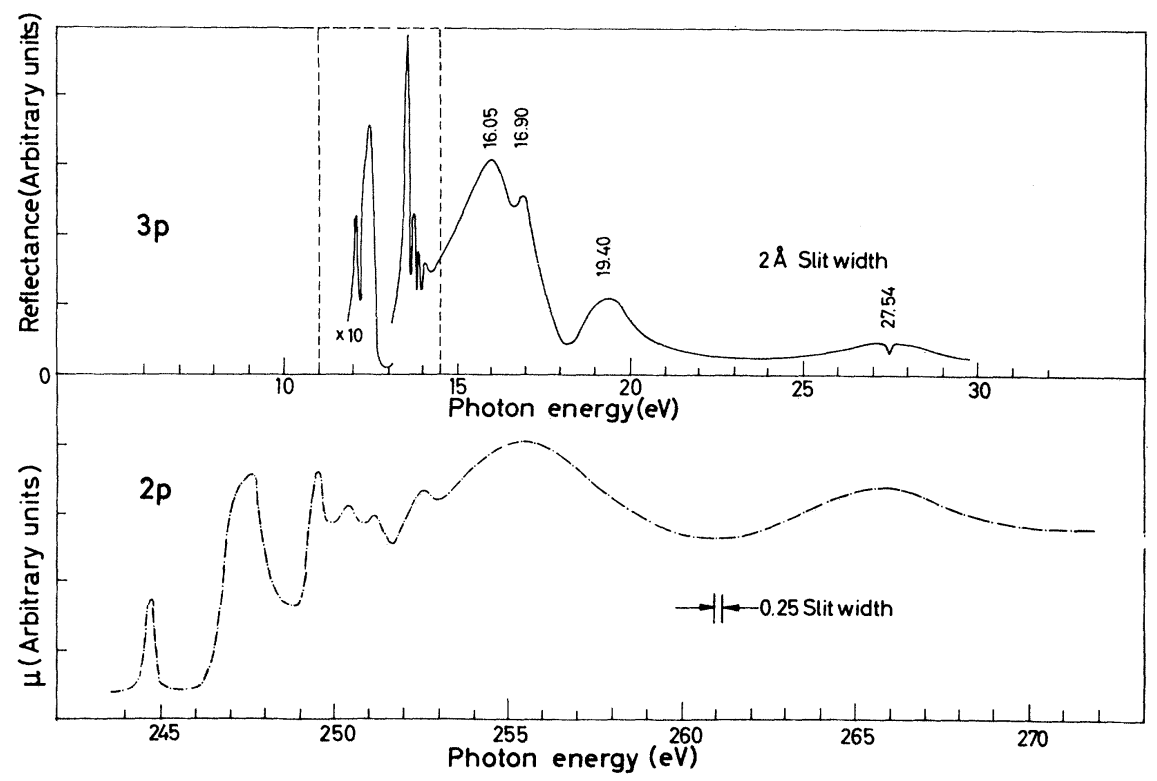

FIG. 2. Reflectance of solid Ar at $20^{\circ} \mathrm{K}$ for an angle of incidence of $15^{\circ}$ between 11 and $30 \mathrm{eV}$ (solid line). Absorption coefficient of solid $\mathrm{Ar}$ at $15^{\circ} \mathrm{K}$ around $250 \mathrm{eV}$ (dashed line). 
surements. Figure 1 shows in an extended scale the fine structure up to $14 \mathrm{eV}$ together with results obtained earlier by Baldini ${ }^{2}$ and by Bostanjoglo and Schmidt. ${ }^{3}$ The reflectance is given in arbitrary units. The internal consistency of the relative heights of the different peaks is better than $5 \%$. While the spectral distribution of the light emerging from the exit slit of the monochromator was taken into account, no absolute calibration was made during the measurements. A rough estimate based on the $\mathrm{KCl}$ reflectivity at room temperature ${ }^{7}$ gave about $70 \%$ for the reflectivity of the highest peak at $12.5 \mathrm{eV}$, but this value may be incorrect by a factor of 2 .

At $12.10 \mathrm{eV}$ we see the first peak in very good agreement with the other authors (Fig. 1).2,3 The second peak is relatively broad. The pronounced shoulder at $12.35 \mathrm{eV}$ coincides with Baldini's peak at $12.3 \mathrm{eV},{ }^{2}$ the maximum at $12.50 \mathrm{eV}$ with the energy-loss maximum of Bostanjoglo and Schmidt $^{3}$ at $12.46 \mathrm{eV}$. Other sharp peaks up to $14 \mathrm{eV}$ can be clearly resolved.

Figure 2 shows broader peaks occuring at higher energies. At $27.5 \mathrm{eV}$ a minimum in the reflectivity curve can be seen, which agrees in its energy position with a "window" line, found in the absorption of solid Ar. ${ }^{8}$

We ascribe the sharp peaks in the reflectance below $14.5 \mathrm{eV}$ to two exciton series, namely two Rydberg series from the spin-orbit-split valence band to the bottom of the conduction band at $\Gamma_{1}{ }^{9,10}$ Our assignments for the different peaks to members of the two Rydberg series, according to the equation ${ }^{11} E=E_{0}-G / n^{2}$, is given in Table I, where $E$ is the peak energy, $E_{0}$ the series limit, $G$ the binding energy, and $n$ the quantum number. As the first exciton may not necessarily fit into the Rydberg formula, ${ }^{11} E_{0}$ and $G$ have been evaluated from the $n=2$ and $n=3$ members. Re-evaluation of the $n=1$ exciton from these values gives an energy $0.25 \mathrm{eV}$ below the experimental values. This, on the other hand, explains the disagreement of our $G$ and $E_{0}$ values with those of other authors, ${ }^{2-4}$ as they were only able to calculate their values from the $n=1$ and $n=2$ excitons.

The two $n=1$ excitons show a temperature dependence: The peak at 12.35-12.5 eV shows slightly varying ratios of the contribution of the two parts, and the peak at $12.1 \mathrm{eV}$ splits into two. A detailed investigation of these effects is in progress.

The structures above $14.5 \mathrm{eV}$ (Fig. 2, upper part) indicate the onset of interband transitions, but no simple correlation with singularities in
Table I. Energy positions and identifications of the exciton peaks.

\begin{tabular}{ccc}
\hline $\begin{array}{c}\text { Peak energy } \\
(\mathrm{eV})\end{array}$ & $\Gamma\left(\frac{3}{2}\right)$ series & $\Gamma\left(\frac{1}{2}\right)$ series \\
\hline 12.10 & $n=1$ & $n=1$ \\
12.35 & & \\
12.50 & $n=2$ & $n=2$ \\
13.58 & $n=3$ & $n=3$ \\
13.75 & $n=4$ & \\
13.90 & $2.30 \mathrm{eV}$ & $2.06 \mathrm{eV}$ \\
14.03 & $14.16 \mathrm{eV}$ & $14.25 \mathrm{eV}$ \\
14.09 & & \\
$G$ & $E_{0}$ & \\
\hline
\end{tabular}

the conduction-band structure obtained by Mattheis ${ }^{10}$ seems possible.

The lower part of Fig. 2 shows for comparison the absorption coefficient due to $2 p$ transitions in solid Ar around $250 \mathrm{eV} .^{12}$ There is an obvious similarity between $3 p$ and $2 p$ transitions. Both spectra begin with a sharp line followed by a broad asymmetric one, and by several weaker peaks. If one attributes also the first line of the $2 p$ absorption spectrum to an exciton series, one is led to assume much larger binding energies for $2 p$ excitons than for $3 p$ excitons. The highenergy parts of the spectra, consisting presumably of broad maxima due to transitions into the conduction-band continuum, reflect further similarity. A conversion of the reflectance data into $\epsilon_{2}$ by a Kramers-Kronig analysis should allow a more precise discussion.

We want to thank the directors of our Institutes for their continuing support.

\footnotetext{
*Work supported by the Deutsche Forschungsgemeinschaft.

${ }^{1}$ J. R. Nelson and P. L. Hartmann, Bull. Am. Phys. Soc. 4, 371 (1959).

${ }^{2}$ G. Baldini, Phys. Rev. 128, 1562 (1962).

${ }^{3}$ O. Bostanjoglo and L. Schmidt, Phys. Letters 22, 130 (1966).

${ }^{4}$ See also: K. Moorjani, Phys. Letters 23, 652 (1966),

${ }^{5}$ R. Haensel and C. Kunz, Z. Angew. Phys. 23, 276 (1967).

${ }^{6} \mathrm{M}$. Skibowski and W. Steinmann, J. Opt. Soc. Am. 57, 112 (1967).

${ }^{7}$ D. Blechschmidt, R. Klucker, and M. Skibowski, Deutsches Elektronen-Synchrotron Report No。DESY 69/27, 1969 (to be published).

${ }^{8}$ R. Haensel, G. Keitel, C. Kunz, and P. Schreiber, to be published.

${ }^{9}$ R. S. Knox and F. Bassani, Phys. Rev. 124, 652
} 
(1961).

${ }^{10}$ L. F. Mattheis, Phys. Rev. 133 , A1399 (1964).

${ }^{11}$ R. S. Knox, The Theory of Excitons (Academic
Press, Inc., New York, 1963).

${ }^{12}$ R. Haensel, G. Keitel, C. Kunz, P. Schreiber, and B. Sonntag, to be published.

\title{
MULTIPLE-SCATTERING DESCRIPTION OF INTENSITY PROFILES OBSERVED IN LOW-ENERGY ELECTRON DIFFRACTION FROM SOLIDS*
}

\begin{abstract}
C. B. Duke
Department of Physics, Materials Research Laboratory, and Coordinated Science Laboratory, University of Illinois, Urbana, Illinois 61801, and General Electric Research and Development Center, Schenectady, New York 12301

and

C. W. Tucker, Jr.

General Electric Research and Development Center, Schenectady, New York 12301

(Received 12 August 1969)

The dependence on beam energy and lattice geometry of the intensity of electrons diffracted from surfaces is evaluated using the inelastic-collision model. At energies below that of the second primary Bragg peak, qualitative features of the intensity profiles are related to the lattice geometry. The quantitative features depend sensitively on the scattering from the surface layer. The first detailed interpretation of such profiles is proposed for the (100) face of tungsten.
\end{abstract}

In recent work we have presented a heuristic derivation, ${ }^{1}$ a perturbation-theory analysis, ${ }^{2}$ and a matrix-inversion analysis ${ }^{3}$ of the inelastic-collision model for the evaluation of the intensity versus energy profiles of low-energy electron beams diffracted from crystal surfaces. In this Letter we summarize the major results of our analysis and note their consequences for the interpretation of experimental data.

Interest in this topic is due to the fact that despite numerous experimental measurements of intensity profiles since $1927^{4}$ and extensive theoretical studies of the effects of multiple scattering,,$^{2-14}$ no satisfactory description of very lowenergy $(E \lesssim 100 \mathrm{eV}$ ) experimental data has been achieved. Our results, presented in Refs. 1-3 and herein, constitute the first systematic discussion of the influence of electron damping and lattice geometry on the predictions of multiplescattering models. We also present herein the first detailed interpretation of experimental intensity profiles in the energy region of the first Bragg peak. The only other such interpretation is that of Hirabayashi, ${ }^{14}$ which adequately describes the observed profiles of carbon exclusively at higher energies where only primary Bragg peaks occur. Earlier attempted interpretations ${ }^{15-17}$ were based on a Darwin kinematical model in which the predicted scattering intensity is not simply related to a solution of the Schröd- inger equation describing the electronic motion. Our analysis also reveals the critical importance for the interpretation of low-energy electron-diffraction (LEED) profiles both of strong inelastic-collision damping and of the electronic inequivalence of the "surface" and "bulk" layers of even a chemically clean, unreconstructed surface. Both of these effects usually have been regarded as "unimportant" in the current theoretical literature ${ }^{7-13,18}$ on multiple-scattering descriptions of LEED from clean, unreconstructed surfaces.

The essential concept underlying the inelasticcollision model ${ }^{1}$ is that the damping of the elastic wave field of an incident electron, due to its excitation of plasmons and incoherent electronhole pairs, is the dominant feature of its motion in a solid which restricts its (elastic) penetration into the solid to a depth of about 5-10 $\AA$. In this limit, ${ }^{1,19}$ the energy widths and maximum intensities of the diffraction peaks are determined primarily by the damping length rather than by the lattice potential of the solid, and the location of the peaks depends primarily on the geometry of the lattice. The analytical formulation of the inelastic-collision model is achieved by using a propagator formalism ${ }^{13}$ to describe the multiple scattering by the lattice. The new feature of the model is the use of electron propagators associated with a (uniform) interacting electron fluid, ${ }^{20}$ 